\title{
The Efficacy of Chitosan Hemostatic Pad on Hemostatic Function in Patients Undergoing Cardiac Catheterization: A Systematic Review and Meta-Analysis
}

\author{
Ya-Ting Lin, MsN, ${ }^{1,2}$ Chia-Jung Hsieh, $\mathrm{PhD},{ }^{1}$ Li-Yen Lee, $\mathrm{PhD}^{3}$ \\ ${ }^{1}$ School of Nursing, College of Nursing, National Taipei University of Nursing and Health Sciences, Taiwan, R.O.C; ${ }^{2}$ St. Mary's \\ Junior College of Medicine, Nursing and Management, Taiwan, R.O.C.; ${ }^{3}$ Cardinal Tien Junior of Healthcare and Management, \\ Taiwan, R.O.C.
}

\section{ABSTRACT}

Background: Chitin is a nitrogen-containing polysaccharide that can promote wound healing and stop bleeding. This paper investigates the effects of the addition of a chitin hemostatic patch on the time to arterial hemostasis, bleeding time, and reduction of the risk of bleeding and hematoma in patients undergoing cardiac catheterization.

Methods: Databases were searched for published clinical studies. The subjects were patients who received cardiac catheterization and had a chitin hemostatic patch added at the site of arterial puncture, while the control group received routine hemostatic treatment. The research quality was evaluated using the Cochrane risk-of-bias tool, version 2.0, and the meta-analysis was carried out using RevMan software.

Results: After searching literature databases, five randomized controlled trials were retrieved and included in the metaanalysis. The results showed that adding a chitin hemostatic patch could shorten the time to arterial hemostasis in patients, who received cardiac catheterization (Std. Mean Difference, $-0.58 ; P<.001)$. In the subgroup analysis, the grouped effect of the chitin hemostatic patch on the bleeding time showed that the bleeding time was not significantly shortened after adding a chitin hemostatic patch in patients in the experimental group (RR, 0.78). At the same time, this measure did not significantly reduce the risk of arterial bleeding (RR, 0.49) or hematoma (RR, 0.73).

Conclusions: The results of the meta-analysis showed that adding a chitin hemostatic patch at the site of arterial puncture in patients undergoing cardiac catheterization significantly reduced the time to hemostasis, but did not significantly reduce the incidence of bleeding and hematoma.

Received May 27, 2021; accepted fune 28, 2021.

Correspondence: Chia-fung Hsieh, PbD, RN, Associate Professor, School of Nursing, College of Nursing, National Taipei University of Nursing and Health Sciences, No. 365, Ming-Te Road, Peitou District, Taipei 11219, Taiwan, ROC, Telephone 886 (2) 2822-7101-3135, Fax +886-2-28206729 (e-mail: chiajung@ntunhs.edu.tw).

\section{INTRODUCTION}

Ischemic heart disease is one of the most common causes of death globally, and a study predicted that by 2020 , the death toll from cardiovascular disease would account for one-third of the global death toll [World Health Organization 2019]. Epidemiological data related to cardiovascular diseases show that smoking, lack of activity and exercise, poor diet and weight control, and other health factors, such as poor control of cholesterol, blood pressure, and blood glucose, often cause cardiovascular health hazards [Bittner 2019]. In addition, international research on the medical cost burden of cardiovascular diseases also shows that this burden has been continuously increasing; for example, the annual direct and indirect costs to people with cardiovascular diseases in the United States are estimated to be about USD \$351.2 billion. From 2014 to 2015, cardiovascular diseases and stroke accounted for $14 \%$ of the total medical expenditure. Among the five common cardiovascular treatment trends for this disease [Benjamin 2019], the cardiac catheterization has gained acceptance the fastest and has become the most common treatment method in the last 20 years, due to its advantages, such as high safety, no need for surgery, short hospital stay, and less pain for patients [Benjamin 2019]. It has even replaced traditional surgical treatment in many aspects and has become a mainstream treatment for many cardiovascular diseases. Moreover, with this cardiovascular treatment, standardized operative procedures can be established, and in-hospital treatment routines can be simplified, effectively reducing the number of days in the hospital, complications, and medical costs through the management of clinical protocols.

Hemostasis after cardiac catheterization is uncertain because of different arterial puncture sites, and seriously affects the treatment outcome and prognosis. Usually, after cardiac catheterization, $56 \%$ of the patients need approximately $10-15 \mathrm{~min}$ for hemostasis at the site of arterial puncture. The average compression time when using an arterial hemostasis compression plate is $>3 \mathrm{~h}$ [Dai 2015; Arbel 2011] and vascular complications, such as arterial bleeding (7-20\%) or hematoma (3-21\%), are common [Dai 2015; Arbel 2011; Mamas 2014; Chugh 2015; Kang 2017; Kikkert 2014; Nguyen 2007]. Conventionally, in this clinical medical procedure, complete arterial hemostasis is achieved by applying manual pressure or by using a vascular closure device [Rao 2016]. 
However, in one clinical case, acute complete transverse tearing of the femoral artery due to asymptomatic arteriosclerosis disease occurred, leading to death several hours after transfemoral cardiac catheterization [D'Ovidio 2016]. To stop the bleeding from the radial artery, a manually pressed wrist band is used as an alternative method [Rao 2016]. According to clinical research, compared with femoral artery puncture, in radial artery puncture, the time to arterial hemostasis is 13 min less, time to mobilization is shorter, recovery comfort is higher, and fewer days of hospital stay are needed [Rao 2016; Mitchell 2012]. In addition, a clinical study showed that the use of transradial cardiac catheterization can prevent the increased cost of vascular or bleeding complications, as compared with transfemoral cardiac catheterization, it has lower cost and fewer associated complications, including vascular complications, making it an economically favorable strategy [Applegate 2013]. However, radial artery occlusion is the most common complication of transradial cardiac catheterization, with an incidence rate of about $0.8-30 \%$, and is attributed to acute thrombosis and intimal hyperplasia, due to vascular endothelial injury. Although arterial compression can achieve hemostasis, it causes blood flow blockage and thrombosis, eventually leading to arterial obstruction. Therefore, it is necessary to use adequate heparin and patent hemostasis measures to prevent this complication [Goswami 2016]. The latter prevents bleeding at the puncture site, while maintaining the antegrade blood flow in the radial artery [Wagener 2015; Wilson 2017].

In recent years, chitin, a nitrogen-containing polysaccharide, has been used as a medical dressing in innovative treatment practices. It can accelerate the healing of wounds and attract the negatively charged platelets in humans. Chitosan is a chitin derivative with many special biological functions, and it is commonly used to produce chitin hemostatic patches, which are used as dressing materials. Research on chitosan nanoparticles revealed that it promotes blood clot formation and shortens the time of blood loss. The relatively short fibrin and plasma clotting times, as well as the shortest whole blood clotting time associated with the use of chitin, may be due to the fact that it provides more contact probability when platelet concentration increases, which promotes platelet aggregation, accelerates the coagulation reaction, and also achieves a rapid hemostatic effect [Chen 2010]. Chitosan itself is a natural alkaline polysaccharide, and as a dressing material, it is expected to provide a moist wound environment, resist bacteria, and improve healing. In addition, solid chitosan can increase platelet adhesion, which is more conducive to hemostasis. It is considered an ideal material to promote wound healing and has become a newly-emerging medical dressing in recent years [Khan 2019; Yang 2008]. Chitin hemostatic dressing has been used in transmaxillary sinus dental surgery [Malmquist 2008], treatment after sinus surgery [Athanasiadis 2009; Valentine 2010; Chung 2016], in other non-vital organs, for hemostasis of vascular access after kidney dialysis [Bachtell 2006; Suzuki 2013], and in patients with acute traumatic hemorrhage [Nct 2017]. Research has shown that the time to hemostasis can be reduced to $<5$ min after using a chitin dressing, and a metaanalysis on the use of chitin dressings after sinus surgery showed that chitin dressings can promote hemostasis and reduce adhesion [Zhou 2017].

Clinical trials have shown that the combination of professional skills with the development of hemostatic consumables will help improve the quality of care provided by medical teams. In recent years, this dressing has been applied to important vital organs, such as the heart and blood vessels. Randomized controlled trials (RCTs) involving the use of chitin dressings for patients after cardiac catheterization have investigated the time to hemostasis in the radial or femoral artery and the risk of vascular complications [Dai 2015; Arbel 2011; Kang 2017; Nguyen 2007; Roberts 2018], but the results have not been verified by a systematic literature review or meta-analysis. This study aimed to explore the effects of adding a chitin hemostatic patch on the time to arterial hemostasis and bleeding time, as well as on reducing the risk of bleeding and hematoma in patients receiving cardiac catheterization through a systematic literature review and empirical meta-analysis. The hypothesis of this study was that adding a chitin hemostatic patch to the site of arterial puncture can significantly shorten the time to hemostasis and decrease the incidence of bleeding or hematoma complications in patients undergoing cardiac catheterization.

\section{METHODS}

\section{Search strategy and selection criteria:}

For this meta-analysis, literature search and screening were carried out according to the standard process guidelines for meta-analysis proposed by Preferred Reporting Items for Systematic Reviews and Meta-Analyses (PRISMA) in 2009. A systematic literature search was conducted in the CINAHL, PubMed, The Cochrane Library, EMBASE, and Google Scholar databases with PICO keywords (Cardiac catheterization OR Percutaneous coronary intervention OR Coronary Artery Disease OR Percutaneous Coronary Revascularizations) AND (chitin OR Chitosan) AND (Hemostasis OR Stanch bleeding), and the reference lists of these studies manually were searched. The literature inclusion criteria were as follows: (1) the subjects should be patients who received transradial or transfemoral cardiac catheterization; (2) the intervention measure should be adding a chitin hemostatic dressing at the radial or femoral artery puncture site; (3) the primary or secondary outcomes should include the time to arterial hemostasis and occurrence of vascular bleeding or hematoma events. Articles were excluded if the studies: (1) were not randomized controlled clinical trials, (2) involved animal trials, and (3) involved coronary stenting. No limitation on years was set for the literature database search. (The systematic review was registered in PROSPERO (CRD42020166590). As a result, a total of five articles satisfied the standard literature searching and screening processes, which are as shown in Figure 1. 


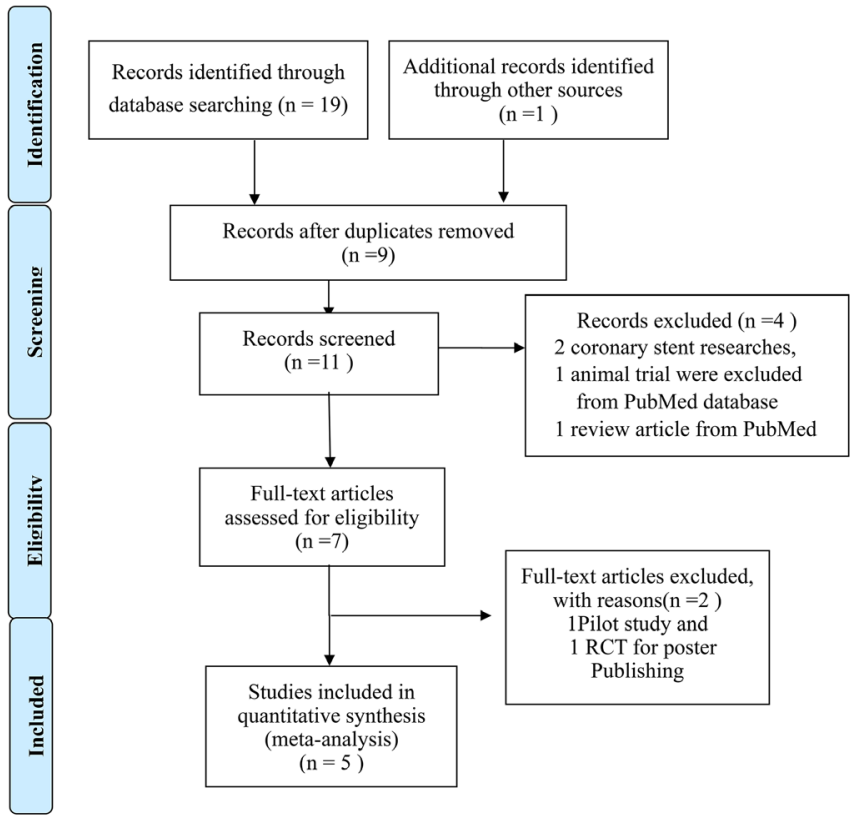

Figure 1. Flow diagram of study searching and selection process

\section{Data extraction and synthesis:}

The first author, year, number of samples, basic attributes of patients, puncture site for cardiac catheterization, average dose of heparin, mean activated coagulation time (ACT), chitin dressing product, time to hemostasis, bleeding and hematoma events, and other reported study data were collected from the included studies. This meta-analysis mainly extracted the above results and further analyzed the time to hemostasis at the site of arterial puncture after a chitin hemostatic patch was used for the patients. The secondary outcomes analyzed were bleeding and hematoma events.

\section{Quality assessment and statistical analysis:}

In this study, two independent reviewers (the first and second authors) commented on the research quality based on the second edition of the research deviation risk assessment method published by the Cochrane Statistical Methods Group and if their opinions differed, the three authors would resolve them through discussion. The results of the time to hemostasis, bleeding time, and bleeding or hematoma events were extracted from published clinical trials and analyzed using Review Manager (RevMan) 5.4 software (The Cochrane Collaboration's software for preparing and maintaining Cochrane reviews). In this meta-analysis, there were data on time to hemostasis with different arterial puncture sites, chitin dressings, and study groups. Considering the heterogeneity of the research designs, the random effects model was adopted for data analysis to avoid underestimating the variability between treatments [Higgins 2003; Hwu 2014].

\section{RESULTS}

\section{Review of literature screening results and research results in each article:}

A total of 20 articles were retrieved after the database search. Of these, 15 articles from different databases were

Table 1. Characteristics of the selected randomized controlled trials

\begin{tabular}{|c|c|c|c|c|c|}
\hline $\begin{array}{l}\text { Kang et al. } \\
2017\end{array}$ & $\begin{array}{l}\text { Open Label RCT }(2: 1) \\
\qquad E G=59 \text { CG }=36\end{array}$ & $\geq 18 y$ TRA & $\begin{array}{l}\text { Combination of Chitosan } \\
\text { based pad and Compres- } \\
\text { sion devices }\end{array}$ & Compression devices alone & $\begin{array}{c}\text { Time to Hemostasis: } \\
\text { Category } \leq 5 \mathrm{~min}, 6-10 \mathrm{~min} \text {, } \\
11-20,>20 \mathrm{~min} \text { Vessel } \\
\text { complication }\end{array}$ \\
\hline $\begin{array}{l}\text { Dai et al. } \\
2015\end{array}$ & $\begin{array}{l}\text { Open Label RCT }(1: 1) \\
\qquad E G=300 \quad C G=300\end{array}$ & $\geq 18 y$ TRA & $\begin{array}{l}\text { Using a chitosan-based pad } \\
\text { (Anscare, Daxon, Taoyuan, } \\
\text { Taiwan) (CS group) con- } \\
\text { stant compression } 10 \text { min } \\
\text { plus elastic bandage }\end{array}$ & $\begin{array}{c}\text { Radial compression device (TR } \\
\text { Band, Terumo Medical, Tokyo, } \\
\text { Japan) (CD group) alone } 8+8 \mathrm{ml} \\
\text { air sac }\end{array}$ & $\begin{array}{l}\text { Time of Compression } \\
\text { Bleeding Radial Artery } \\
\text { Occlusion }\end{array}$ \\
\hline $\begin{array}{l}\text { Arbel et al. } \\
2011\end{array}$ & $\begin{array}{c}\text { Open Label } \mathrm{RCT} \text { EG }=70 \\
\mathrm{CG}=66\end{array}$ & $18 y-80 y$ TFA & $\begin{array}{c}\text { The HemCon® pad (Hem- } \\
\text { Con Medical Technologies, } \\
\text { USA) was applied manually } \\
\text { for } 5 \text { minutes + pressure } \\
\text { bandage }\end{array}$ & $\begin{array}{c}\text { Regular pad was applied manu- } \\
\text { ally for } 5 \text { minutes plus pressure } \\
\text { bandage }\end{array}$ & $\begin{array}{l}\text { Time to hemostasis } \\
\text { Category } 5 \mathrm{~min}, 10 \mathrm{~min}, \\
>15 \mathrm{~min} \text { Time to Hemostasis } \\
\text { continued data Vascular } \\
\text { complication }\end{array}$ \\
\hline $\begin{array}{l}\text { Amoozgar et } \\
\text { al. } 2020\end{array}$ & $\mathrm{RCT} E \mathrm{EG}=30 \mathrm{CG}=30$ & TFA $<16 \mathrm{Y} / \mathrm{O}$ & Celox powder $2 \mathrm{gm}$ & Manual pressure & $\begin{array}{c}\text { Time to Hemostasis Case } 17.2 \\
\pm 6.7 \text { Control } 20.3 \pm 13.6\end{array}$ \\
\hline
\end{tabular}


Table 2. Research data of the selected randomized controlled trials

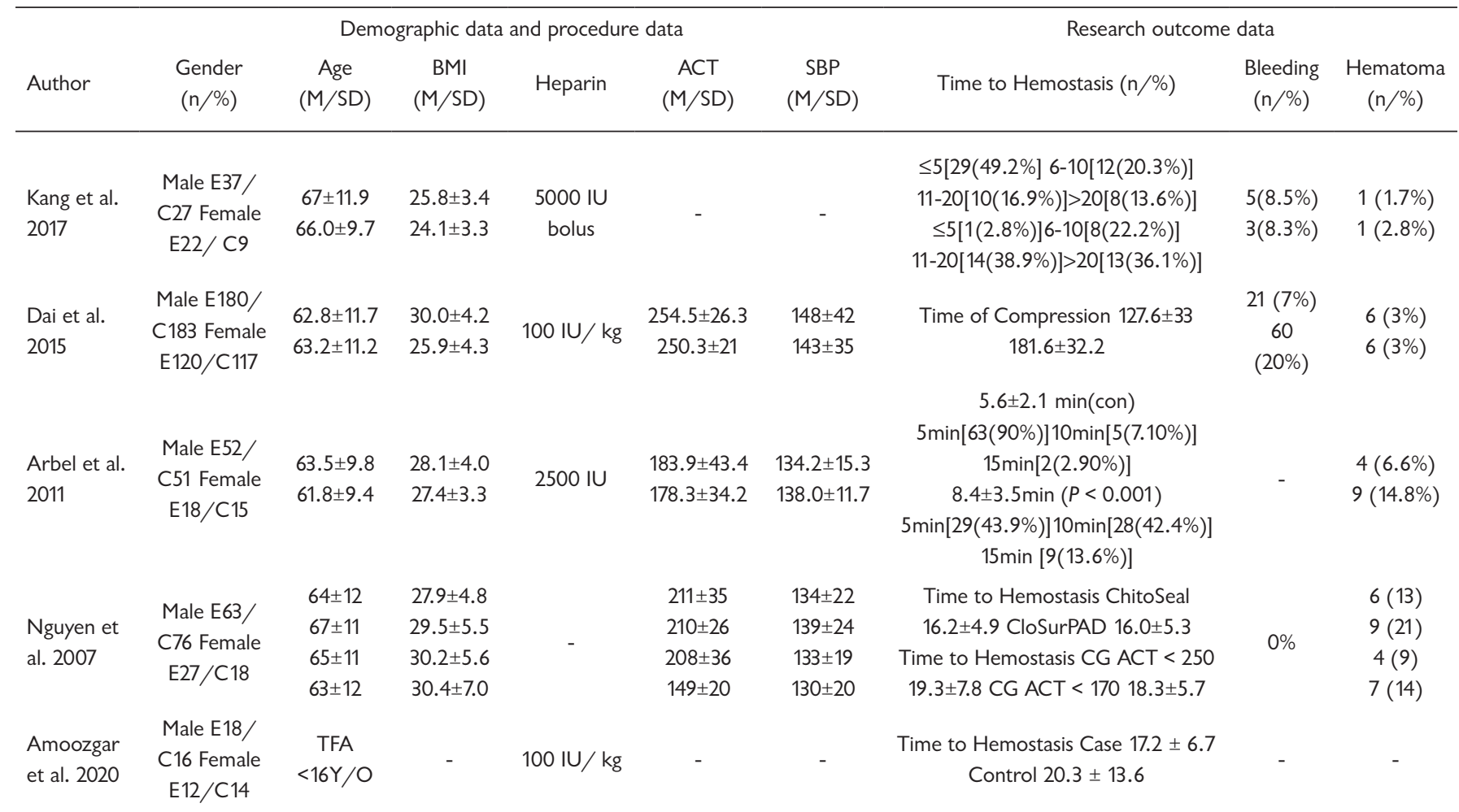

Table 3. Methodological Quality assessment of the included trails (Version 2 of the Cochrane risk-of-bias assessment tool)

\begin{tabular}{|c|c|c|c|c|c|}
\hline Domains & Kang et al. 2017 & Dai et al. 2015 & Arbel et al. 2011 & Nguyen et al. 2007 & Amoozgar et al. 2020 \\
\hline Bias arising from the randomization process & Low risk & Low risk & Some concern & Some concern & Some concern \\
\hline Bias due to missing outcome data & Low risk & Low risk & Low risk & Low risk & Low risk \\
\hline Bias in measurement of the outcome & Low risk & Low risk & Low risk & Low risk & Low risk \\
\hline Overall bias & Low risk & Low risk & Some concern & Some concern & Some concern \\
\hline
\end{tabular}

excluded as repeated studies (nine articles), literature review (one article), studies on drug-eluting stents (two articles), animal experiment (one article), and quasi-experimental study and poster publication (two articles). Finally, the remaining five qualified articles were included in the evaluation of research quality. The research quality evaluation results of all clinical trials were consistent between the two reviewers. All the recruited cases in these studies received cardiac catheterization. The study design included explicit inclusion (cardiac catheterization through radial or femoral artery puncture) and exclusion (abnormal coagulation function) criteria. The experimental group (EG), in which a chitin hemostatic patch (Anscare, HemCom, ChitoSeal, CloSur, or Celox Powder) was added to for hemostasis, and the control group (CG), which was treated by hand compression or radial artery compression device (TR Band), were compared to evaluate the arterial hemostatic effect, including the time to arterial hemostasis, bleeding time, and bleeding or hematoma events outcome variables. The overall basic attributes and intervention measures of the studies are listed in Tables 1 and 2.

\section{Time to hemostasis at the arterial puncture site:}

Review of the results of the five studies showed that Arbel et al. [Arbel 2011] and Nguyen et al. [Nguyen 2007] measured the continuous time to hemostasis at the arterial puncture sites and found that the time to hemostasis in the EG was significantly shorter than in the CG (mean=5.6 \pm 2.1 , $P<0.001$, ChitoSeal $=16.2 \pm 4.9$, CloSurPAD $=16.0 \pm 5.3, P=$ 0.005 ). However, in a study by Amoozgar et al. [Amoozgar 2020], there was no significant difference in the time to arterial hemostasis between the two groups (EG:17.2, CG:20.3, $P=0.77)$. Other studies classified the time to hemostasis as 


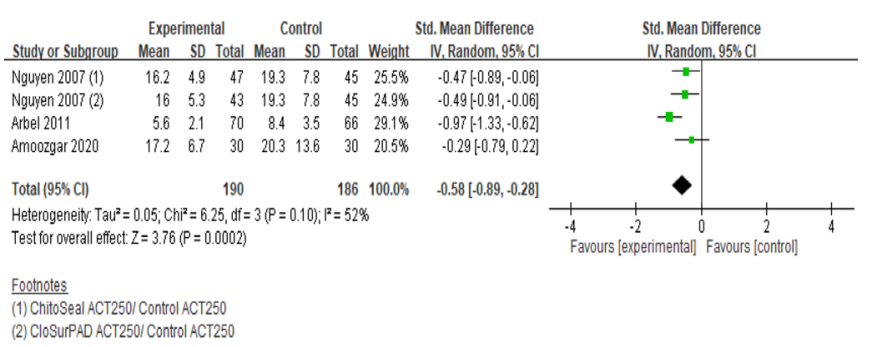

Figure 2. Forest plot for the meta-analysis of time to Hemostasis (continue data)

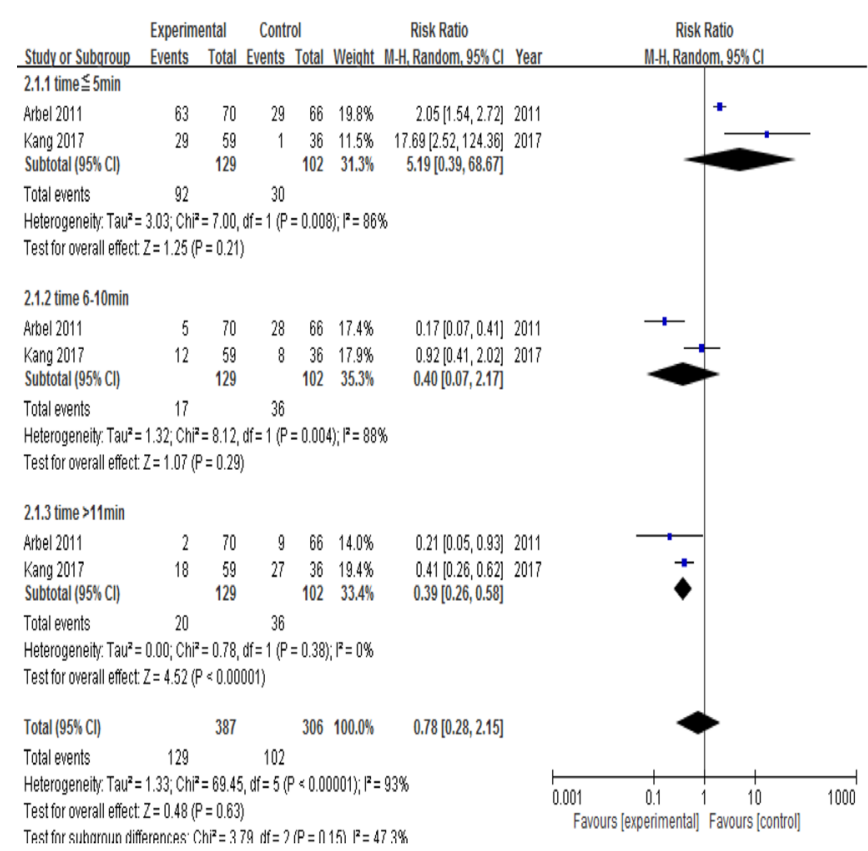

Figure 3. Forest plot for the meta-analysis of bleeding time (categorical data)

categorical data. In a study by Kang et al. [Kang 2017], hemostasis was achieved within $10 \mathrm{~min}$ in $69 \%$ of patients with a chitin hemostatic patch, compared with only $25 \%$ in the CG. In a study by Arbel et al. [Arbel 2011], hemostasis was achieved within $10 \mathrm{~min}$ in $97 \%$ of patients in the EG and $86 \%$ in the CG, with a statistically significant difference between the two groups.

Bleeding and hematoma events at the artery puncture site:

Kang et al. [Kang 2017] showed that the incidence of bleeding events in the two groups was similar (EG:8.5\%, CG:8.3\%), while Dai et al. [Dai 2015] showed that the relative risk of bleeding events in EG was $47.5 \%$, the absolute risk reduction was $9.5 \%$, and the confidence interval was narrow, which showed that the research results were accurate.

In addition, regarding the incidence of hematoma events at the arterial puncture sites, the studies by Kang et al. [Kang 2017] and Arbel et al. [Arbel 2011] showed that the relative risk of hematoma in EG was reduced by $39 \%$ and $58 \%$,

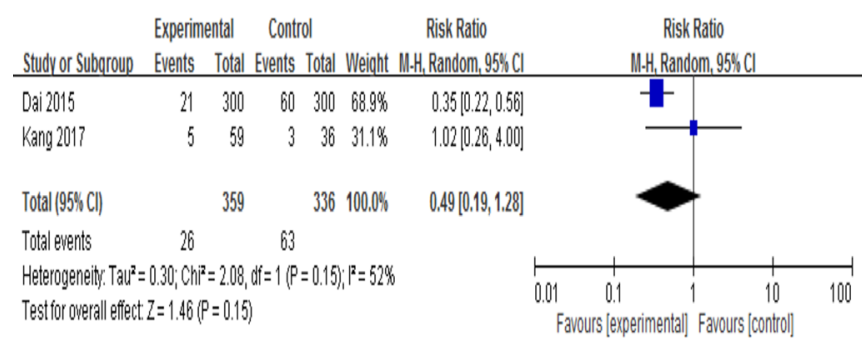

Figure 4. Forest plot for the meta-analysis of bleeding event

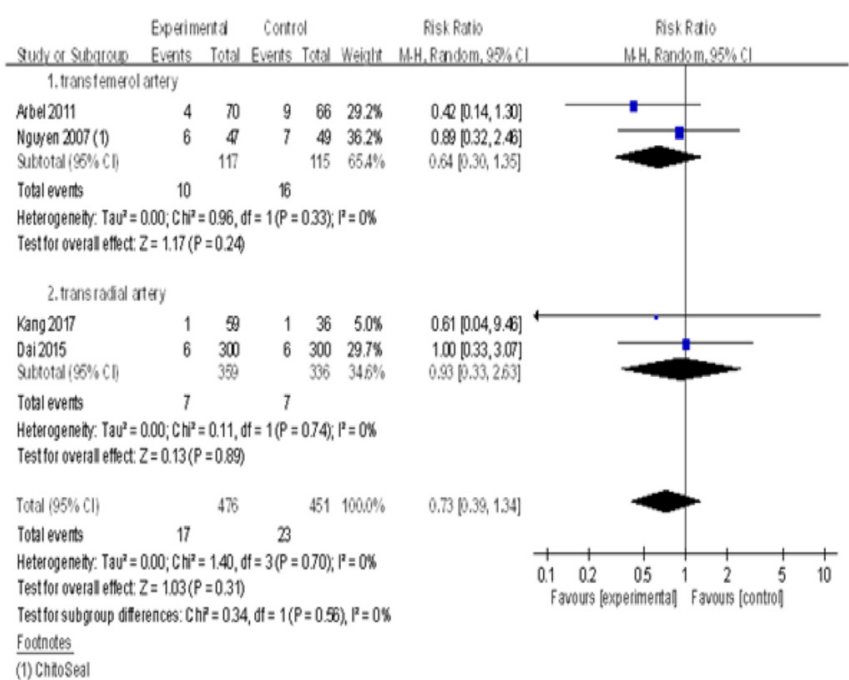

Figure 5. Forest plot for the meta-analysis of hematoma events

respectively; the absolute risk reduction in EG was 1.1\% and $7.9 \%$, respectively. The results of these two clinical trials showed that although the relative and absolute risks of hematoma complications were reduced after adding a chitin hemostatic patch at the site of arterial puncture for cardiac catheterization, the confidence interval was wide and there was no significant difference; thus, the research results were not accurate. However, the results of another study by Nguyen et al. [Nguyen 2007] were contrary to the above studies: The relative and absolute risks of hematoma in EG decreased (4.36\% and $3.9 \%$, respectively). In addition, in a study by Dai et al. [Dai 2015] because the number of cases enrolled $(N=300)$ and the number of hematoma events were the same $(\mathrm{N}=6)$ in $\mathrm{EG}$ and CG, the risk of hematoma could not be compared. Thus, the above five clinical studies show that the time to hemostasis, bleeding time, and bleeding and hematoma complications after cardiac catheterization were not the same in patients with and without a chitin hemostatic patch and require meta-analysis.

Overall effect size of chitin hemostatic patch on arterial hemostatic function:

\section{Overall effect of adding chitin hemostatic patch on time to hemostasis:}

Arbel et al. [Arbel 2011], Nguyen et al. [Nguyen 2007], and Amoozgar et al. [Amoozgar 2020] collected continuous 
data on the time to hemostasis at arterial puncture sites, and according to our meta-analysis, the heterogeneity between the studies was high $\left(\mathrm{I}^{2}=52 \%\right)$. The overall effect showed that the diamond was biased toward the EG with added chitin hemostatic patches, indicating that the time to hemostasis at arterial puncture sites was significantly reduced (Std. mean difference, -0.58 ; $95 \% \mathrm{CI},-0.89$ to $-0.28, \mathrm{Z}=3.76, P<$ 0.001). (Figure 2)

\section{Grouped effect of adding a chitin hemostatic patch on the bleeding time:}

Among the five RCTs, only two studies, one by Kang et al. [Kang 2017] and another by Arbel et al. [Arbel 2011] analyzed the time to hemostasis (time to hemostasis, categorized into $\leq 5,6$ to $10, \geq 11-20$ or $\geq 20$ minutes). After regrouping the research data (categorized into $\leq 5,6$ to $10, \geq 11$ minutes), the results of the meta-analysis showed that the heterogeneity between the two studies was high, and the overall effect showed that the diamond was biased toward the EG, but the $95 \%$ confidence interval of RR (RR, $0.78 ; 95 \%$ CI, 0.28 to $2.15, Z=0.48, P=0.63$ ) crossed 1 (the line of no effect), which showed that the time to hemostasis of patients in EG with a chitin hemostatic patch at the arterial puncture site was not significantly shortened. (Figure 3)

\section{Results of bleeding risk reduction by adding chitin hemostatic patch:}

Only two of the included studies, one by Dai et al. [Dai 2015] and another by Kang et al. [Kang 2017], collected data on vascular bleeding, and this meta-analysis showed moderate heterogeneity. The results of the analysis of arterial bleeding events in the EG with a chitin hemostatic patch showed that the diamond of overall effect crossed the line of no effect $(\mathrm{RR}=0.49 ; 95 \% \mathrm{CI}, 0.19$ to $1.28, \mathrm{Z}=1.46, P=0.15)$. Thus, it revealed that adding a chitin hemostatic patch in the EG did not significantly reduce the risk of bleeding events after cardiac catheterization. The results of the meta-analysis are shown in Figure 4.

\section{Results of hematoma risk reduction by adding a chitin hemostatic patch:}

All the studies included in the meta-analysis showed high homogeneity $\left(\mathrm{I}^{2}=0 \%\right)$, but because only five studies were combined for analysis, the number of studies was insufficient, and it was impossible to confirm whether heterogeneity actually did not exist. In terms of arterial hematoma events when a chitin hemostatic patch was added in the EG, the analysis results showed that the diamond of overall effect crossed the line of no effect $(\mathrm{RR}=0.73 ; 95 \%$ CI, 0.39 to $1.34, Z=1.03, P=0.31)$. Dividing the studies into transradial and transfemoral groups for sub-group analysis revealed that the effect size, as shown by the diamond, was biased toward the EG, but it still crossed the line of no effect, showing that adding a chitin hemostatic patch did not significantly reduce hematoma formation at arterial puncture sites. The results of the meta-analysis are shown in Figure 5.
DISCUSSION

\section{Effect of chitin hemostatic patches on arterial hemostasis:}

This meta-analysis explores the time to arterial hemostasis after adding a chitin hemostatic patch post-cardiac catheterization treatment through arterial puncture, which is widely applied clinically. The results showed that, in terms of the overall effect on the time to hemostasis after adding a chitin hemostatic patch, the diamond was biased toward the EG with added chitin hemostatic patches, and the time to hemostasis at arterial puncture sites was shortened by $2.8-3.1$ min (Std. mean difference, $-0.58 ; 95 \%$ CI, -0.89 to -0.28 , $Z=3.76, P<0.001)$, which is similar to the results of rapid hemostasis when chitin dressings were used in other medical applications [Athanasiadis 2009; Valentine 2010; Chung 2016]. For example, research on hemostatic treatment after sinus surgery revealed that chitosan gel can achieve rapid hemostasis after injury, with an average time to hemostasis of 2 min [Valentine 2010].

Moreover, in a study on hemostatic treatment after tooth extraction, all patients on oral anticoagulant treatment could achieve hemostatsis at the site of transmaxillary sinus dental surgery in $<1$ min after applying a HemCon dental dressing [Malmquist 2008]. In addition, the average time to hemostasis of the vascular access after kidney dialysis was 15-60 min, and when a HemCon bandage was used, hemostasis could be achieved within $2 \mathrm{~min}$ in $97.3 \%$ of kidney dialysis sessions, which reduced the long bleeding time at the puncture site and the use of vascular compression band, thus reducing thrombosis [Bachtell 2006; Suzuki 2013]. Lastly, in 133 patients with acute scalp injury, hemostasis could be achieved in $<5 \mathrm{~min}$ (4.68 $\pm 1.04 \mathrm{~min})$ when using an Axiostat dressing [Nct 2017].

The subgroup analysis on arterial bleeding time showed that the number of patients in whom hemostasis took $>11$ min was significantly less in EG with added chitin hemostatic patches than in CG, but EG did not show a significant advantage in terms of the number of cases who achieved hemostasis within $5 \mathrm{~min}$ and those who achieved hemostasis between $6 \mathrm{~min}$ and $10 \mathrm{~min}$. Because there were as few as only two articles included in the bleeding time analysis, the arterial puncture sites also were different, and the meta-analysis showed high heterogeneity. Therefore, it needs to be verified in future studies.

\section{Effect of chitin hemostatic patches on bleeding and hematoma risk reduction:}

Heparin is one of the most commonly used anticoagulants, and its main purpose is to reduce the risk of thrombosis in cardiac catheterization. However, research has shown that higher doses of heparin are associated with more bleeding complications, and when activated clotting time, ACT approaches $365 \mathrm{~s}$, the risk of bleeding increases linearly, which in turn increases the morbidity and mortality due to vascular complications [Lee 2015; Brener 2004]. In this meta-analysis, a chitin dressing was used for patients in EG, but it did not significantly reduce the incidence of bleeding 
and hematoma. This might be related to the chitin dressing products used in the analyzed studies, because the molecular weights of chitosan in the dressings used in the four studies [Dai 2015; Arbel 2011; Kang 2017; Nguyen 2007] were different, and the measures, such as the use of rotating compression devices and hand compression hemostasis, also were different. In addition, the dose of heparin varied from study to study. Kang et al. [Kang 2017] and Dai et al. [Dai 2015] used 5000IU direct injection or $100 \mathrm{IU} / \mathrm{Kg}$ dose of heparin, while Arbel et al. [Arbel 2011] used a lower dose of heparin with an average of about 2500IU, and the ACT of all patients in the studies was $<250 \mathrm{~s}$; thus, it could not be ascertained whether adding a chitin hemostatic dressing at the arterial puncture site in patients receiving cardiac catheterization can effectively reduce arterial bleeding or hematoma. Therefore, further RCTs are needed to verify this.

\section{Evaluation of research quality:}

The research quality of the literature included in the analysis was evaluated, according to the second edition of the research deviation risk assessment method published by the Cochrane Statistical Methods Group in 2019 [Sterne 2019]. The first dimension of this assessment analyzes the quality of the randomization process in the research. All the five articles were RCTs. However, Arbel et al. [Arbel 2011], Nguyen et al. [Nguyen 2007], and Amoozgar et al. [Amoozgar 2020] did not clearly explain whether their assignment sequences were blinded, which led to some doubts regarding the research quality. In addition to the blindness in the randomization method and grouping, the first aspect also emphasizes interpretation of articles when there are differences in baseline values. The revision additionally requires that contingent baseline differences will not lead to deviation risk. For example, the randomization process was not explained in the study by Nguyen et al. [Nguyen 2007], and there were significant gender differences among different groups. However, because the sample distribution ratio was close to the characteristics of the population, it was judged that there was no research bias, while the characteristics of patients were not significantly different between the two groups in the other studies.

The second dimension investigates whether deviation from the intervention measures creates bias. The second edition of the research deviation risk assessment method published by the Cochrane Statistical Methods Group mentions that if there is no deviation from the expected intervention due to the test environment, the deviation risk of an open-label trial may be low. Three studies were open-label trials, which showed that it was difficult to implement blindness because of the appearance (special color) of the chitin dressing used in patients undergoing cardiac catheterization. However, the hemostatic effect in patients is not affected by consciousness or human intervention, so the research risk assessment indicated a low risk.

Further, the third dimension evaluates the errors caused by missing result data. The result data of all subjects were presented. The evidence showed that there was no error in the results, and the risk assessment was low risk. The fourth dimension measures the errors caused by the test results.
All the studies investigated the arterial hemostatic function of chitin dressings, and their measurement indicators were time to hemostasis or events of vascular complications, which were in line with their operational definition. All the studies adopted a single-center design, and the study by Dai et al. [Dai 2015] had a single data collector. Although the assignment sequence was not blinded, it did not affect the judgment of the results, and the research was assessed as high quality. Finally, the fifth dimension evaluates the errors caused by selective reporting of test results. The study by Arbel et al. [Arbel 2011] used continuous data for the time to arterial hemostasis, and at the same time converted them to time categorial data at different time cut-off points, which was regarded as reasonable data analysis with low risk in research quality evaluation.

At last, the overall research risk assessment showed that the studies of Kang et al. [Kang 2017] and Dai et al. [Dai 2015] both had low risk, while those of Arbel et al. [Arbel 2011], Nguyen et al. [Nguyen 2007], and Amoozgar et al. [Amoozgar 2020] were rated as having some doubts in research quality because the articles do not specify whether the assignment sequence was blinded. The research quality risk assessment for the studies with doubts in research quality is shown in Table 3. (Table 3) This meta-analysis used the updated version of Cochrane risk-of-bias tool, and its assessment results can provide reference.

\section{CONCLUSIONS}

Heparin is widely used in cardiac catheterization to prevent arterial obstruction caused by thrombi. Literature verification shows that non-occlusive hemostasis is an independent predictor for preventing arterial obstruction in patients undergoing cardiac catheterization. Its purpose is to maintain antegrade blood flow in the radial artery and prevent bleeding at the puncture site. Therefore, the primary hemostatic function of chitin plays an important role after intravenous injection of a heparin bolus for cardiac catheterization. The results of this meta-analysis showed that in patients undergoing cardiac catheterization, adding a chitin hemostatic patch at the arterial puncture site, could significantly reduce the time to hemostasis to within $5 \mathrm{~min}$. However, the subgroup analysis showed that there was no difference in grouped effect on bleeding time after adding a chitin hemostatic patch. At the same time, this measure did not significantly reduce the risk of hematoma or arterial bleeding. However, the continuous improvement in the expertise of cardiologists, and the development of hemostatic consumables for arterial puncture sites, can shorten the time to arterial hemostasis, help to reduce the vascular complications and consequent consumption of medical recourses, and improve the care quality of medical teams. Therefore, it is suggested that a clinical cost-benefit analysis of this dressing should be carried out in the future so as to provide a reference for practical application selection. 


\section{ACKNOWLEDGEMENTS}

We acknowledge that all authors listed meet the authorship criteria, according to the latest guidelines of the International Committee of Medical Journal Editors. And all authors read and approved the final manuscript.

\section{REFERENCES}

Amoozgar H, Abtahi S, Edraki MR, Mohammadi H, Ajami GH, Mehdizadegan N, Keshavarz K, Moradi P, Shabanpuor-Haghighi S, Zafari T. 2020. Effect of Celox ${ }^{\circledR}$ Powder on Initial Hemostasis After Cardiac Catheterization in Pediatric Patients with Congenital Heart Disease: A Prospective Study. Iranian Journal of Pediatrics. 30:1-6.

Applegate R, Sacrinty M, Schafer P, Smith J, Gandhi S, Kutcher M, Santos R, Cecile A, Little W. 2013. Cost effectiveness of radial access for diagnostic cardiac catheterization and coronary intervention. Catheterization and cardiovascular interventions : official journal of the Society for Cardiac Angiography \& Interventions. 82:E375-84.

Arbel J, Rozenbaum E, Reges O, Neuman Y, Levi A, Erel J, Haskia AR, Caneti M, Sherf M, Mosseri M. 2011. USage of chitosan for Femoral (USF) haemostasis after percutaneous procedures: a comparative open label study. EuroIntervention. 6:1104-9.

Athanasiadis T. 2009. The effect of topical antifibrinolytics and a novel chitosan gel on haemostasis and wound healing in endoscopic sinus surgery.

Bachtell N, Goodell T, Grunkemeier G, Jin R, Gregory K. 2006. Treatment of dialysis access puncture wound bleeding with chitosan dressings. Dialysis \& Transplantation. 35:672-681.

Bittner VA. 2019. The New 2019 ACC/AHA Guideline on the Primary Prevention of Cardiovascular Disease. Circulation. 140.

Benjamin EJ, Muntner P, Alonso A, Bittencourt MS, Callaway CW, Carson AP, Chamberlain AM, Chang AR, Cheng S, Das SR, et al. 2019. Heart Disease and Stroke Statistics-2019 Update: A Report From the American Heart Association. Circulation. 139:e56-e528.

Brener SJ, Moliterno DJ, Lincoff AM, Steinhubl SR, Wolski KE, Topol EJ, Brener SJ, Moliterno DJ, Lincoff AM, Steinhubl SR, et al. 2004. Relationship between activated clotting time and ischemic or hemorrhagic complications: analysis of 4 recent randomized clinical trials of percutaneous coronary intervention. Circulation. 110:994-998.

Chen HS. 2010. The application of chitosan nanoparticles in hemostasis [dissertation]. Douliou, Yulin: National Yunlin University of Science and Technology.

Chugh SK, Chugh Y, Chugh S. 2015. How to tackle complications in radial procedures: Tip and tricks. Indian heart journal. 67:275-81.

Chung YJ, An SY, Yeon JY, Shim WS, Mo JH. 2016. Effect of a chitosan gel on hemostasis and prevention of adhesion after endoscopic sinus surgery. Clinical and Experimental Otorhinolaryngology. 9:143-149.

D'Ovidio C, Sablone S, Carnevale A. 2016. Death Due to an Unusual Angio-Seal-Related Complication: Case Report and Literature Review. Journal of forensic sciences. 61:1364-8.

Dai N, Xu DC, Hou L, Peng WH, Wei YD, Xu YW. 2015. A comparison of 2 devices for radial artery hemostasis after transradial coronary intervention. Journal of Cardiovascular Nursing. 30:192-6.

Goswami R, Oliphant CS, Youssef H, Morsy M, Khouzam RN. 2016.
Radial Artery Occlusion After Cardiac Catheterization: Significance, Risk Factors, and Management. Current problems in cardiology. 41:214-227.

Higgins JP, Thompson SG, Deeks JJ, Altman DG. 2003. Measuring inconsistency in meta-analyses. BMJ. 327:557-60.

Hwu YJ and Lin FY. 2014. Effectiveness of propolis on oral health: a meta-analysis. The Journal of Nursing Research. 22:221-9.

Kang SH, Han D, Kim S, Yoon CH, Park JJ, Suh JW, Cho YS, Youn TJ, Chae IH. 2017. Hemostasis pad combined with compression device after transradial coronary procedures: A randomized controlled trial. PLoS One. 12:e0181099.

Khan MA and Mujahid M. 2019. A review on recent advances in chitosan based composite for hemostatic dressings. International journal of biological macromolecules. 124:138-147.

Kikkert WJ, Delewi R, Ouweneel DM, van Nes SH, Vis MM, Baan J, Jr., Koch KT, Dangas GD, Mehran R, de Winter RJ, Peters RJ, Piek JJ, Tijssen JG, Henriques JP. 2014. Prognostic value of access site and nonaccess site bleeding after percutaneous coronary intervention: a cohort study in ST-segment elevation myocardial infarction and comprehensive metaanalysis. JACC Cardiovascular interventions. 7:622-30.

Lee MS and Kong J. 2015. Heparin: Physiology, Pharmacology, and Clinical Application. Reviews in Cardiovascular Medicine. 16:189-199.

Malmquist JP, Clemens SC, Oien HJ, Wilson SL. 2008. Hemostasis of oral surgery wounds with the HemCon Dental Dressing. Journal of Oral and Maxillofacial Surgery. 66:1177-1183.

Mamas MA, Fraser DG, Ratib K, Fath-Ordoubadi F, El-Omar M, Nolan J, Neyses L. 2014. Minimising radial injury: prevention is better than cure. EuroIntervention. 10:824-32.

Mitchell MD, Hong JA, Lee BY, Umscheid CA, Bartsch SM, Don CW. 2012. Systematic review and cost-benefit analysis of radial artery access for coronary angiography and intervention. Circulation Cardiovascular quality and outcomes. 5:454-62.

Nct. 2017. Studying Safety \& Efficacy of Axiostat ${ }^{\circledR}$ Dressing in Acute Hemorrhage Due to Trauma-Comparative Study. https:// clinicaltrialsgov/show/NCT03035695.

Nguyen N, Hasan S, Caufield L, Ling FS, Narins CR. 2007. Randomized controlled trial of topical hemostasis pad use for achieving vascular hemostasis following percutaneous coronary intervention. Catheterization and cardiovascular interventions : official journal of the Society for Cardiac Angiography \& Interventions. 69:801-7.

Rao SV and Stone GW. 2016. Arterial access and arteriotomy site closure devices. Nature reviews Cardiology. 13:641-650.

Roberts JS, Niu J, Pastor-Cervantes JA. 2018. Comparison of hemostasis times with a chitosan-based hemostatic pad (Clo-Sur(Plus) Radial) vs mechanical compression (TR Band(R)) following transradial access: A pilot study. Cardiovascular revascularization medicine : including molecular interventions.

Sterne JAC, Savovic J, Page MJ, Elbers RG, Blencowe NS, Boutron I, Cates CJ, Cheng HY, Corbett MS, Eldridge SM, et al. 2019. RoB 2: a revised tool for assessing risk of bias in randomised trials. BMJ. 366:14898.

Suzuki S, Shibata K, Kuji T, Kawata S, Koguchi N, Nishihara M, Satta H, Toya Y, Umemura S. 2013. Randomized trial comparing newchitosan-based bandage with kaltostat hemostatic dressing to control bleeding from hemodialysis puncture site. Nephrology Dialysis Transplantation. 28:i228-i229.

Valentine R, Athanasiadis T, Moratti S, Hanton L, Robinson S, Wormald PJ. 2010. The efficacy of a novel chitosan gel on hemostasis and wound 
healing after endoscopic sinus surgery. American Journal of Rhinology and Allergy. 24:70-75.

Wagener JF and Rao SV. 2015. Radial artery occlusion after transradial approach to cardiac catheterization. Current atherosclerosis reports. $17: 489$.

Wilson SJ, Mitchell A, Gray TJM, Loh HJ, Cruden NL. 2017. Patent haemostasis prevents radial artery occlusion in patients with an acute coronary syndrome. International Journal of Cardiology. 240:78-81.

World Health Organization. 2019. The top 10 causes of death. WHO Web site. https://www.who.int/news-room/fact-sheets/detail/the-top10-causes-of-death. Accessed December 21.
Yan YH, Chen Y, Kung CM, Peng LJ. 2011. Continuous quality improvement of nursing care: case study of a clinical pathway revision for cardiac catheterization. The Journal of Nursing Research. 2011;19:181-9.

Yang J, Tian F, Wang Z, Wang Q, Zeng YJ, Chen SQ. 2008. Effect of chitosan molecular weight and deacetylation degree on hemostasis. Journal of biomedical materials research Part B, Applied biomaterials. 84:131-7.

Zhou JC, Zhang JJ, Zhang W, Ke ZY, Zhang B. 2017. Efficacy of chitosan dressing on endoscopic sinus surgery: a systematic review and metaanalysis. European Archives of Oto-Rhino-Laryngology. 274:3269-3274. 